\title{
EL ROSTRO DE LA VIOLENCIA SOCIAL Y ESTRUCTURAL: LA DELINCUENCIA Y LA POBREZA COMO EXPRESIONES DISTINTAS DE UNA VULNERABILIDAD COMÚN
}

\section{THE FACE OF SOCIAL AND STRUCTURAL VIOLENCE: CRIME AND POVERTY AS DIFFERENT EXPRESSIONS OF A COMMON VULNERABILITY}

\author{
Andrés Castillo Vargas* \\ Xiomara Castro Chaves ${ }^{* *}$
}

\begin{abstract}
RESUMEN
El presente artículo pretende analizar de manera general, los cimientos del fenómeno de la violencia social y estructural, en aras de desmitificar cualquier posible relación causal entre pobreza $y$ delincuencia, $y$ procurar su reconocimiento como manifestaciones de un mismo proceso de vulnerabilidad, que aleja cada vez más al ser humano del ejercicio pleno de sus derechos y oportunidades. Producto del recorrido efectuado, se concluye que la idea distorsionada de que las y los delincuentes son personas pobres, invisibiliza la vulnerabilidad social de grupos humanos específicos y lleva a obviar la ocurrencia de determinados delitos. Negando de esta manera, las causas reales de la conducta criminal, las cuales se ubican en el sistema socio económico actual que incrementa las brechas sociales y que carece de fundamentos basados en la expansión de las capacidades y libertades reales de la ciudadanía (Sen, 1985).
\end{abstract}

PALABRAS CLAVE: DELINCUENCIA * POBREZA * VULNERABILIDAD SOCIAL * VIOLENCIA ESTRUCTURAL * ESTRATIFICACIÓN SOCIAL

Instituto de Investigaciones Psicológicas (IIP) de la Universidad de Costa Rica. andres.castillo@ucr.ac.cr
** Asistente de investigación del Instituto de Investigaciones Psicológicas (IIP) de la Universidad de Costa Rica.

xiobs@yahoo.es 


\section{ABSTRACT}

This article aims to analyze in a general manner, the foundation of the social and structural violence phenomenon, in order to demystify any possible causal relationship between poverty and crime, and to seek its recognition as manifestations of the same process of vulnerability, which increasingly removes most human beings from fully exercising their rights and opportunities. As a product of the journey made, it is concluded that the distorted idea that offenders are poor people, obscures the social vulnerability of specific human groups and leads to obviate the occurrence of certain crimes, denying the real causes of criminal behavior, which are found in the current socioeconomic system that increases social gaps and lacks foundations based on the expansion of capabilities and real citizen freedom (Sen, 1985).

KEYWORDS: DELINQUENCY * POVERTY * SOCIAL VULNERABILITY * STRUCTURAL VIOLENCE * SOCIAL STRATIFICATION

\section{INTRODUCCIÓN}

El presente artículo busca reflexionar en torno a la relación existente entre la delincuencia y la pobreza como expresiones de la violencia social y estructural. Para ello, se analizará cómo ambos fenómenos sociales pueden ser vistos como manifestaciones distintas de una vulnerabilidad común, siempre y cuando sean analizados desde una perspectiva social (Martín Baró, 2000), que evidencie la correspondencia existente entre las raíces de la delincuencia y el momento histórico y coyuntural que atraviesa nuestra sociedad, el cual se caracteriza por el aumento vertiginoso de la exclusión de determinados grupos humanos. Ejemplo de ello, es que en Costa Rica existen actualmente 52 distritos con Muy alto rezago social, los cuales son territorios muy extensos y con muy baja densidad poblacional, factor que constituye una limitación para acceder a mejores servicios que les permita alcanzar un mayor desarrollo socioeconómico (González, 2004).

De acuerdo con el Programa de las Naciones Unidas para el Desarrollo, las causas de este aumento en la desigualdad son diversas, no obstante, se vinculan en gran medida con el tipo de crecimiento económico que ha mostrado el país en los últimos años, en donde:

... la brecha salarial entre los trabajadores calificados y los no calificados, así como entre los formales y los informales y agropecuarios aumentó en la presente década respecto a los años noventa, impulsando el aumento en la desigualdad en la distribución del ingreso (PNUD, 2011:11).

Teniendo en consideración este proceso de desigualdad y exclusión social, en este artículo se entenderá por violencia:

... el uso deliberado de la fuerza física, [psicológica y simbólica], ya sea contra uno mismo, otra persona o un grupo o comunidad, que cause o tenga probabilidades de causar lesiones, muerte, daños psicológicos, trastornos del desarrollo o privaciones (OPS, 2002: 5).

De este modo, la violencia simbólica específicamente, será considerada como un tipo de violencia que opera en las esferas comunicativas de la cultura, en sus signos y símbolos, siendo a su vez una de las violencias más sutiles $y$ difíciles de identificar, ya que al presentarse en espacios de representación y comunicación social, ha sido naturalizada tanto por las personas que la reciben como por las personas que la ejercen (Plaza, 2007).

Esta definición brinda una conceptualización ampliada a las significaciones tradicionales referidas a la violencia, haciéndola concordante a los modelos ecológicos de análisis social, los cuales resaltan la interacción de diversos factores (estructurales, políticos, 
económicos, etc) en la conformación o aparición de un fenómeno. En este sentido, de acuerdo con la propuesta de Bronfenbrenner (2002), los fenómenos sociales pueden ser comprendidos con base en sistemas, los cuales se clasifican en ontosistema (esfera individual), microsistema (esfera familiar), ecosistema (esfera comunitaria) y macrosistema (esfera social y estructural); cuya interacción puede dar origen o explicación al por qué de la existencia de diversas manifestaciones culturales.

Esta concepción ecológica de la violencia nos enfrenta a su intrínseca complejidad, dado que las causas de la misma no pueden ubicarse de forma aislada en alguno de los sistemas mencionados anteriormente, sino en la interrelación de los mismos. En esta línea, uno de los elementos que debe considerarse dentro de dicha dinámica es el papel que los medios de comunicación masiva desempeñan como promotores de opinión pública o como perpetuadores de la estigmatización social de determinados grupos humanos.

Es común observar en los medios de comunicación, una gran variedad de noticias referentes a robos, tráfico de drogas, homicidios y otras formas de violencia que hace sentir insegura a la ciudadanía, al mismo tiempo que evidencian cómo estamos siendo azotados/as por una gran "ola de delincuencia" que atenta contra los "valores" y las "buenas costumbres" familiares, convirtiendo a la delincuencia en uno los principales problemas de la sociedad actual. Sin embargo, este manejo realizado por los medios de comunicación y por la sociedad en general, se convierte en un mecanismo de control social que perpetúa una idea distorsionada de cuáles son las causas reales de la delincuencia y del aumento de la violencia en nuestra sociedad, ya que tiende a enfatizar que la pobreza es la causa directa de los delitos.

Al respecto, cabe citar que "la delincuencia es un fenómeno social que depende tanto del tratamiento que recibe en los medios de comunicación como de las políticas públicas diseñadas para su prevención" (Cisneros, 2007: 255). Asimismo, es importante reconocer que el concepto de violencia es mucho más amplio que el concepto de agresión (Martín Baró, 2000) tradicionalmente enfatizado por los medios de comunicación masiva, pues la violencia tiene un carácter histórico-social que la describe, de allí la importancia de entenderla dentro de un contexto sociocultural en específico. Dicha apreciación es reforzada por Sebastian, Oettler y Peetz, los cuales opinan que "hay que interpretar a la violencia y delincuencia como construcciones sociales y no como fenómenos objetivamente existentes" (2007:79).

Así, a pesar de que violencia y agresión tienen una valoración social negativa, es la violencia la que impone a las economías nacionales una gigantesca carga financiera de miles de millones de dólares (OPS, 2002); razón por la cual debe ser prevenida $y$ analizada desde sus raíces y en sus diversas manifestaciones.

Considerando este panorama, los siguientes apartados analizarán la relación existente entre la pobreza y la delincuencia, percibidas como expresiones de la segregación y la exclusión social suscitadas por el sistema económico actual. Con ello, no se pretende justificar la comisión de un delito, sino desmitificar la estigmatización que poseen los grupos en exclusión social, debido a que la criminalización de la pobreza se ha convertido en la justificación cultural por antonomasia, ante el aumento vertiginoso de la delincuencia, haciendo creer erróneamente que la delincuencia es originada directa y exclusivamente por la pobreza. De esta manera, se refuerza la asociación entre pobres $y$ delito como una relación unicausal y determinista, que obvia la responsabilidad del Estado como promotor de novedosas formas de estratificación y exclusión que incrementan la inopia y las privaciones que sufren determinados grupos humanos.

Finalmente, el presente artículo pretende constituirse en un insumo más para que los temas de pobreza $y$ delincuencia también sean abordados desde el punto de vista de las ciencias sociales y no solo desde modelos represivos, estigmatizantes, ahistóricos y reduccionistas.

\section{EL FENÓMENO DE LA VIOLENCIA Y SU VÍNCULO CON LA EXCLUSIÓN Y LA ESTRATIFICACIÓN SOCIAL}

Con el fin de aproximarnos a comprender la pobreza $y$ la delincuencia como manifestaciones 
de una vulnerabilidad común, es necesario describir a grandes rasgos el fenómeno de la violencia social y estructural desde sus orígenes, es decir, aquellos sistemas ideológicos, políticos y económicos que a lo largo de la historia han establecido regímenes socioculturales caracterizados por la desigualdad y exclusión, los cuales aún imperan en la actualidad. Asimismo, en aras de comprender cómo se entreteje este vínculo entre exclusión y delincuencia en nuestro país, debe recurrirse a elementos contextuales y macroeconómicos que han permeado las sociedades latinoamericanas, de los cuales Costa Rica no se encuentra exenta.

Para Martín Baró (2000), la violencia estructural alude al ejercicio de la desigualdad opresiva, legitimada socialmente, que genera un efecto nocivo en la ciudadanía y promueve el establecimiento de una espiral de violencia. De acuerdo con esta premisa, la estratificación social podría considerarse entonces un mecanismo para el mantenimiento de un orden social, producto y reflejo del dominio de una clase social sobre otra, así como, una clara manifestación de la violencia presente en el mismo ordenamiento social. Por ello:

... la estrecha vinculación entre justificación de la violencia e intereses sociales dominantes muestra que, en definitiva, la violencia no es medida por sí misma, sino por sus productos. Se justifica aquella violencia que favorece los propios intereses, lo que, al interior de un orden social establecido, significa el apoyo a los intereses dominantes (Martín Baró, 2000: 377).

En el caso específico de América Latina, la inestabilidad política, la ingobernabilidad $y$ la crisis económica han desencadenado un aumento alarmante en los niveles de violencia en todos los estratos y niveles sociales, rompiendo los lazos de solidaridad $y$ haciendo trizas el tejido social comunitario (Galeano, 1996). Siguiendo a Rodríguez (2004), si bien los modelos de desarrollo actuales adoptaron como principio fundamental la apertura de los mercados al capital transnacional, ello desató un aumento considerable en la desigualdad social estructural y por tanto, de la pobreza. Por tales razones, la pobreza y la violencia se han convertido en ejes principales dentro de las agendas públicas de los países latinoamericanos, siendo dos manifestaciones distintas de un mismo proceso de vulnerabilidad acaecido alrededor del mundo.

Retomando varios de los argumentos esbozados con anterioridad, Basaglia plantea que:

.. la aceptación de esta división como fenómeno natural (la existencia del rico y del pobre como dato irreductible natural en el [ser humano]), comparte una serie de reglas $y$ de instituciones que con la aparente finalidad de resolver las contradicciones naturales, sirve de hecho para mantener la originaria división sobre la que se erige la estructura económicasocial, es decir, sirven al mantenimiento del statu quo (1980: 54).

Por consiguiente, es importante visualizar clara y contundentemente que la violencia que nos aqueja actualmente, no es más que un mecanismo estratégico que promueve la exclusión y desigualdad entre los grupos sociales, promoviendo la "represión de las necesidades reales y por tanto de los derechos humanos en su contenido histórico-social" (Baratta, 1991: 22 ), condenando a los más desposeídos a vivir en un estado de fragilidad, frente a una sociedad cada vez más individualista que privilegia al poderoso y castiga al más pobre, culpabilizando su condición y atribuyéndole una serie de estigmas, que inclusive han llegado a crear un pseudo-vínculo de causalidad entre la pobreza y la delincuencia.

\section{DELINCUENCIA Y POBREZA: EXPRESIONES DISTINTAS DE UNA VULNERABILIDAD COMÚN}

Si se abriera un periódico de circulación nacional y se tratara de poner un rostro a las personas que, de acuerdo con dichos medios, actualmente se encuentran cometiendo delitos e infringiendo la ley; podría percatarse de que comparten características similares, entre ellas: presentan un bajo nivel de escolaridad, tienen 
poco acceso a los servicios sociales y la gran mayoría se encuentra en situación de pobreza. Aunado a ello, Calderón plantea que en el perfil sociológico de los delincuentes destaca que “... su abrumadora mayoría son hombres jóvenes que provienen de los segmentos bajos y que han nacido en una familia numerosa, esto además de presentar una escasa especialización delictiva" (2006: 86). Dichas características permiten pensar que el tipo de delito se relaciona con la posición social que ocupa la persona infractora; es decir, no cualquier persona puede cometer cualquier delito (Calderón, 2006).

Esto no quiere decir que exista una relación causal entre delincuencia y pobreza, más bien la pobreza debe visualizarse como un factor de vulnerabilidad social que incrementa el riesgo de que un grupo importante de la población se vea en la necesidad de realizar actos delictivos como mecanismo de sobrevivencia, pues:

... quienes aceptan y persiguen las metas que promueve la sociedad, pero no disponen de los medios legítimos para alcanzarlas, serán quienes tiendan, en mayor proporción, a cometer actos delictivos; sin embargo, este actuar constituye solo una de las posibles respuestas ante el desfase entre metas y medios (Calderón, 2006: 107).

Por esta razón, el autor identifica dos tipos de infractores: los infractores por necesidad, para quienes "... el delito constituye un medio necesario para sobrevivir, una forma de complementar el ingreso proveniente de otras actividades económicas..." [y los infractores por motivación, quienes] “... ven en el delito una actividad económica que les permitirá superar su situación y alcanzar una movilidad social ascendente" (Calderón, 2006: 111).

Considerando dicha clasificación, ¿cómo podría explicarse la delincuencia de cuello blanco que nos asecha en la actualidad? ¿Acaso los políticos y los grandes ejecutivos que frecuentemente protagonizan actos delictivos, son pobres? Estas y otras interrogantes podrían ayudar a esclarecer el panorama manifiesto en nuestra sociedad, donde la delincuencia no puede ser atribuida a una clase social en particular.
Es por esta razón, que los grupos humanos en exclusión social:

... no se encuentran exentos de protagonizar lamentables acontecimientos que se ven influidos tanto por la percepción pública construida del crimen y del victimario, como por los ajustes económicos producidos por la globalización y sus consecuencias directas en el incremento de la pobreza y la desigual distribución de la riqueza (Cisneros, 2007: 255-256).

En este sentido, de manera tradicional "la pobreza ha sido entendida como carencia de recursos materiales que impiden la satisfacción de las necesidades básicas por la imposibilidad de adquirir los bienes y servicios esenciales" (Rodríguez, 2004: 4). Sin embargo, "además de la privación material, la pobreza comprende dimensiones subjetivas que van más allá de la subsistencia material" (Instituto Interamericano de Derechos Humanos, 2008: 18), ejemplo de ello, es la falta de potencialización de las capacidades de las personas - que si bien- podría estar relacionado con la carencia de ingresos económicos, no debe restringirse a estos (Sáenz, 2009), pero sí debe considerarse una forma de exclusión del ejercicio de sus derechos humanos $y$ por ende, una manifestación de la violencia social y estructural imperante en nuestra sociedad. De esta forma:

... la violencia estructural no se reduce a una inadecuada distribución de los recursos disponibles que impide la satisfacción de las necesidades básicas de las mayorías; la violencia estructural supone además un ordenamiento de esa desigualdad opresiva, mediante una legislación que ampara mecanismos de distribución social de la riqueza y establece una fuerza coactiva para hacerlos respetar (Martín Baró, 2000: 406).

Asimismo, puede ser considerada como "la forma general de la violencia, en cuyo contexto directa o indirectamente encuentran su fuente en gran parte, todas las otras formas de violencia" (Baratta, 1991: 22). 
Ahora bien, retomando estos aportes para analizar con detenimiento la relación entre delincuencia y pobreza, se puede conceptualizar la delincuencia como un fenómeno histórico social, resultante de un modelo de desarrollo excluyente, en el cual “... se justifica el estudio del delito en sus relaciones con la estructura social y económica, pues ello permite evidenciar el carácter social antes que individual de esta problemática" (Calderón, 2006: 84), empero, el Estado y los medios de comunicación masiva continúan haciendo pagar las consecuencias de este fenómeno a personas individuales, obviando que quienes delinquen son instrumentos y víctimas de la misma sociedad que les culpa y castiga sin asumir su responsabilidad directa en este fenómeno.

De acuerdo con Claramunt (2004: 34), esa tendencia a interpretar los problemas sociales como resultado de la dinámica psicológica individual o interpersonal, se denomina Psicologismo, misma que "se deriva del sistema o corriente filosófica denominada individualismo, que considera al individuo particular como unidad o centro de análisis y por tanto, como fundamento y fin del conocimiento", obviando por completo aquellos factores sociales, económicos, culturales y políticos que influyen en el origen y desarrollo de un fenómeno social como lo es la delincuencia.

Por lo tanto, siguiendo esta línea, los únicos/as responsables de cometer actos delictivos serían las mismas personas que los ejercen, de manera que si se "repara" su disfuncionalidad particular e individual, la delincuencia desaparecería (Claramunt, 2004). Esto además de constituir una visión reduccionista y fragmentada de la realidad, aleja a las personas que delinquen de nuevas oportunidades y acceso a los servicios sociales, pues incrementa el estigma de que son personas "locas", "pobres", "vagabundas" y "problemáticas" que lo que únicamente necesitan es "cura" o aislamiento, sin que el Estado asuma su cuota de responsabilidad al respecto.

En palabras de Becker, citado por Calderón:

... el hecho de ser atrapado y etiquetado como desviado (delincuente) tiene consecuencias importantes para la futura participación social y la autoimagen, pues a partir de ese momento el individuo será tratado en forma diferente, pues ha revelado ser de una clase distinta a la que se pensaba (2006: 98).

Así, la misma violencia promovida por el poder social establecido, causa la continua frustración de aspiraciones fundamentales y suscita el cumplimiento de metas a partir de medios éticamente cuestionables para el bien común, haciendo emerger la individualidad como norma, en una lógica que justifica la opresión y en síntesis, la reproducción del ejercicio de la violencia en sus múltiples formas.

La promoción de esta emergente individualidad, puede percibirse en el modelo actual de desarrollo que presenta Costa Rica, el cual se encuentra caracterizado por la globalización, la apertura del mercado, la homogenización y la búsqueda de mejores y más rápidas formas de productividad que conviertan al Estado en un ente mucho más dinámico. Este individualismo va ligado a otro valor característico de las sociedades capitalistas: la exaltación de la competencia, en donde el fin primordial para lograr la satisfacción individual es la competitividad, es decir, aquel tipo de actividad donde el éxito personal requiere la opresión, derrota o exclusión de otro.

Empero, esta sobrevaloración del mercado, de la tecnología y del consumo ha causado grandes transformaciones en las estructuras sociales, las cuales han originado que las y los ciudadanos cambien la manera de ver su propia identidad $y$ de vivir su ciudadanía. Adamson considera que "este momento histórico se caracteriza por una velocidad y magnitud de los cambios tecnológicos, de sentido de normatividades, de reglas de juego que produce una vivencia de torbellino como percepción del contexto y de desamparo como condición subjetiva" (1997: 41). De acuerdo con este autor, anteriormente la identidad de las personas era determinada por distintas esencias históricas como la nacionalidad, ahora la identidad está sujeta a lo que el individuo posea. Retomando sus palabras: "tú eres 
lo que consumes o puedas consumir, esto es lo que te dará identidad, y como lo que consumimos varía, nuestra identidad es inestable".

Por su parte, Fournier argumenta que:

... la necesidad de incrementar la eficiencia y la productividad ha puesto, hoy más que nunca, al dinero y al estatus en una situación de privilegio dentro de nuestro sistema axiológico. Cada vez con mayor intensidad, la capacidad de consumo se constituye en la característica primordial, tanto para valorar a nuestros ciudadanos como para evaluar nuestra propia proximidad a la felicidad $y$ al éxito en la vida. Sin embargo, en un mundo de recursos escasos, las posibilidades reales de lograr este ideal de consumo creciente solo es accesible a una proporción cada vez más reducida de la población, y por el contrario, las grandes mayorías con ingentes esfuerzos apenas logran sobrevivir... Ante esta situación, no debemos extrañarnos de encontrar indicadores que muestren tendencias muy marcadas hacia el individualismo (2000: 152).

Asimismo, el sentido comunitario de las personas ha cambiado, ya no existe interés por los problemas de nuestros vecinos/as, si estos no nos afectan directamente, la corresponsabilidad social hacia ciertos problemas de orden público ha desaparecido y lo que priva es un sentido individualista y salvaje de "sálvese quien pueda".

Como corolario de los argumentos anteriores, Caldeira y López citadas por Juárez, concluyen que en la actualidad:

... persiste una visión de ciudad como espacio de amenaza, donde la criminalidad ha tomado un lugar central..., donde los ciudadanos se preocupan por su integridad física, por los robos, los asaltos $y$ los secuestros, por perder un patrimonio que les ha costado forjar (2006: 46).

Al parecer, la vorágine de la globalización exige la pérdida de las fuerzas gregarias y comunitarias, así como, las actitudes pro sociales y deliberativas que expresan valores cooperativos, evidenciando así, cómo la hegemonía del mercado está rompiendo los vínculos solidarios entre la ciudadanía. Muestra de ello, es que las altas tasas de criminalidad atraen la atención del sector público, mientras que la pobreza es pasada por alto (Giddens, 1992).

Lo anterior también es sostenido por Calderón, quien afirma que:

... durante los últimos años, la situación delictiva del país ha sido uno de los aspectos que más atención ha recibido por parte de la sociedad costarricense. Para los habitantes constituye una de sus mayores preocupaciones, para los partidos políticos un referente ineludible en la búsqueda por captar votos, $y$ para las instituciones vinculadas con éste un desafío de magnitud insospechada (2006: 83).

A manera de ejemplo, las estadísticas del Ministerio de Justicia costarricense, registran que durante el periodo de 1985 y el 2003, el país experimentó un aumento del 114,2\% en delitos como: agresión, violación, hurto, robo, estafa e infracción a la Ley de Psicotrópicos, convirtiéndose la inseguridad en una de las mayores preocupaciones para la ciudadanía (Programa de las Naciones Unidas para el Desarrollo, 2005).

No obstante, la solución brindada a estos problemas de inseguridad ciudadana se ha caracterizado por el aumento vertiginoso de mecanismos de represión y control social, dejando a un lado la creación de políticas públicas y culturales preventivas del delito, por lo cual esta situación, aunada al desarraigo comunitario e inexistencia de conciencia pro social en la ciudadanía, han ocasionado que las brechas sociales se incrementen, aumentando la inequidad entre la población y por consiguiente, apareciendo "nuevos pobres".

Según Gutiérrez (2004), los períodos de crecimiento de la pobreza en Costa Rica se han encargado de convertir a un vulnerable (casi pobre) en pobre, e inclusive no han logrado alejar a los no pobres de la pobreza, perpetuando la exclusión social en la cual otros/as ya se encontraban y transformando su condición de pobreza en miseria extrema. Tales 
circunstancias parecieran confirmar, cómo "entre los factores que entrañan un riesgo de que estallen conflictos violentos figuran las desigualdades sociales, caracterizadas por grandes diferencias en la distribución y el acceso a los recursos" (OPS, 2002: 5). Justamente:

... el tema controversial es que el "combate" a la pobreza, como se le ha denominado, forma parte integral del ciclo político y por tanto está fuera de la racionalidad tecnocrática. Pero también y he aquí su principal limitación, responde a diseños de corto plazo organizados para contener resistencias sociales y captar afiliación partidaria pero no para ofrecer umbrales de superación, en el largo plazo, de los niveles de pobreza (Sojo, 1997: 29).

Al respecto, Adamson (1997) considera que la actual política mundial de exclusión y disgregación, produce la segregación de un grupo importante de nuestra sociedad, perfilando así, un modelo de desarrollo en el que sus miembros no están ya vinculados como una sociedad de semejantes. En la actualidad, de acuerdo con la perspectiva de dicho autor, se quiere instaurar un modelo de exclusión que originará el exilio de una gran parte de la población del ejercicio pleno de su ciudadanía y garantías fundamentales.

Dentro de este panorama, los sectores vulnerables de nuestra sociedad son etiquetados negativamente sin tomar en cuenta aquellos factores sociales, económicos y culturales que influyen de manera directa en sus acciones, concentrando con ello la atención social en una visión reduccionista, alejada de cualquier comprensión ecológica que integre la interacción de diversos componentes intervinientes.

Ahora bien, tomando en consideración una postura ecológica que integre aspectos macro, meso y micro, dicha vulnerabilidad social puede ser entendida:

... como fragilidad e indefensión ante cambios originados en el entorno, como el desamparo institucional desde el
Estado que no contribuye a fortalecer ni cuidar sistemáticamente de sus ciudadanos; como debilidad interna para afrontar concretamente los cambios necesarios del individuo $\mathrm{u}$ hogar para aprovechar el conjunto de oportunidades que se le presenta; [o] como inseguridad permanente que paraliza, incapacita $y$ desmotiva la posibilidad de pensar en estrategias $y$ actuar a futuro para lograr mejores niveles de bienestar (Busso, 2001: 8).

Como puede notarse, esta definición enfatiza cómo la vulnerabilidad social va más allá de las privaciones materiales, pues refiere también a la privación en la capacidad de resiliencia, lo cual debilita los recursos personales para enfrentar $y$ actuar ante diferentes situaciones presentes en el entorno inmediato (Hernández, 2011), paralizando de una u otra forma la capacidad de acción y re-acción de los individuos.

Esta vulnerabilidad social se acrecenta con la pobreza y es el origen de un proceso de estigmatización utilizado por el Estado y por los medios de comunicación, para generalizar $y$ reforzar los prejuicios y estereotipos atribuidos a las personas pobres, haciendo pensar a la sociedad en general que el problema de la delincuencia es la pobreza y no el modelo social imperante. En este sentido, se promueven ideas distorsionadas que hacen pensar a la ciudadanía que "ser joven, pertenecer a un determinado grupo social $y$ vivir en un determinado espacio es equivalente a ser peligroso, drogadicto, marihuano o violento" (Cisneros, 2007: 256).

Esta mitificación social de la pobreza origina que determinados grupos humanos interioricen una visión negativa de sí mismos, la cual es reforzada culturalmente, obviando un sin número de actos delictivos cometidos por personas con gran poder adquisitivo.

Se habla entonces de ciertas condiciones de exclusión social y segregación que originan la noción equivocada de que son solamente las personas pobres quienes delinquen, obviando las razones macro estructurales que se encuentran detrás de la delincuencia. Ampliando esta 
idea, Sojo (1997: 60) considera que "la pobreza es una condición que degrada a quienes la padecen, hiere la autoestima y el espíritu de lucha, es cada vez más difícil de superar intergeneracionalmente y se constituye sobre la base de comparaciones denigrantes", denunciando como determinados grupos humanos conservan en su vulnerabilidad social, otras caras del ser humano, como lo son: el sufrimiento, la impotencia, la opresión y la necesidad de una existencia que no sea estigmatizada (Basaglia: 1980).

Ante lo expuesto, cabe la pregunta de ¿cuál es la reacción de la sociedad costarricense frente a este fenómeno?

La respuesta a dicha interrogante, se puede ubicar en el estudio de la criminología de la reacción social, la cual engloba a la criminología interaccionista, la cual se interesa en investigar la reacción de la sociedad ante las conductas, ya sea creando normas penales, reprimiendo los hechos punibles o estigmatizando, señalando y/o etiquetando a las personas que delinquen (Quinney, 1975).

En palabras de Sebastian, Oettler y Peetz:

... la forma en que una sociedad percibe $y$ reacciona ante la violencia y la delincuencia depende más de los procesos dentro de esta sociedad para "negociar" la definición y el sentido de la violencia y la delincuencia —es decir, depende más del discurso sobre estos fenómenosque de los actos de violencia o delincuencia como tales (2007: 83).

Nuestra sociedad ha reaccionado diferencialmente a este fenómeno, por un lado, están las personas que cuestionan directamente al Estado y su responsabilidad ante los actos delictivos, por otro lado, están las y los ciudadanos que no se involucran en el tema $y$ finalmente, está el grupo que considera que la solución a la delincuencia es la coerción y la fuerza, leyes más duras y mecanismos de control social mucho más fuertes y si estos no son eficaces, es válido ejercer la justicia por su propia cuenta.

De esta forma, "se considera normal la tortura del delincuente común, o de quién tenga cara de..." (Galeano, 1996: 154), olvidando que la delincuencia representa una creación cultural con una característica particular: la de ser exigible y juzgable mediante la amenaza de sanción, según las normas establecidas por una justicia de índole pro social y consensuada. La delincuencia, desde este punto de vista, es una situación general que obedece a tendencias individuales, sociales, económicas, históricas y políticas que deben ser solucionadas por vías democráticas de administración de la justicia $y$ no por mecanismos violentos, de coerción o estigmatización.

En síntesis, las líneas anteriores pretenden brindar una primera aproximación desmitificadora sobre la supuesta relación causal entre la pobreza $y$ delincuencia, de manera que a través del recorrido reflexivo en torno al modelo económico que impera en la actualidad y las diversas manifestaciones de la violencia estructural, se pueda reconocer que la pobreza no es una causa directa de la delincuencia, sino un factor que vulnerabiliza a las personas en una sociedad injusta, que les aleja del ejercicio de una ciudadanía plena, pues no brinda ni garantiza oportunidades equitativas de acceso a los servicios sociales y al ejercicio de los derechos humanos en general.

Tal panorama, constituye un desafío no solo para el Estado costarricense, sino también para los medios de comunicación y la sociedad civil, pues además de políticas públicas que ataquen la raíz del fenómeno, se requieren políticas culturales que den fe sobre un cambio en la forma de pensar y actuar de las personas.

\section{REFLEXIONES FINALES}

Para concluir esta reflexión en torno a la delincuencia y la pobreza, entendidas como expresiones de la violencia social y estructural, es relevante enfatizar la importancia de comprender esta relación desde un enfoque ecológico, pues la asociación entre pobres $y$ delito no es una relación unicausal ni determinista, sino una manifestación cultural producto de la interacción entre factores individuales, familiares, comunitarios y sociales (Bronfebrenner, 2002). En este sentido, se insiste en visualizar la pobreza como un factor de vulnerabilidad 
social que aumenta el riesgo de que los grupos humanos más desposeídos sean excluidos de un ejercicio pleno de su ciudadanía y etiquetados como "chivos emisarios" del sistema dominante.

Asimismo, resulta indispensable analizar cómo dentro del contexto y modelo económico actual, la delincuencia crece mucho más rápido que los recursos que desarrollamos para combatirla, a la vez que el Estado demuestra dificultades en asumir proactivamente su cuota de responsabilidad en la proliferación de distintas manifestaciones de violencia e inequidad. En esta línea, Galeano argumenta que "los gobiernos latinoamericanos confiesan de alguna manera su impotencia, pero el poder jamás confiesa que está en guerra contra los pobres que genera, en pleno combate contra las consecuencias de sus propios actos" (1996: 156).

Es impostergable que el Estado impacte sobre la pobreza que experimentan las personas, este impacto solamente es positivo si logramos eliminarla progresivamente; también debe impulsar la creación de mecanismos de prevención de la delincuencia, mediante el fomento de factores protectores, mejorar la reinserción social de las personas que han cometido un delito, crear mecanismos de control social respetuosos de los derechos humanos y sobre todo, impactar sobre la estigmatización que sufren ciertos grupos sociales $y$ devolverles la autoestima e identidad perdidas.

Una forma de cumplir con dichas metas es fomentando el desarrollo de capacidades $y$ libertades por medio de la creación de políticas públicas inclusivas, que remitan a la progresividad de las libertades ciudadanas (Instituto Interamericano de Derechos Humanos, 2008). Sin embargo, dicha tarea también debería ser asumida por los medios de comunicación masiva y las organizaciones de la sociedad civil, responsables del control social y —a la fecha- de un análisis distorsionado del fenómeno de la violencia, debido al desconocimiento y manipulación de la información. Por lo tanto, el compromiso de estas entidades debería reflejarse en la promoción de cambios en función del bienestar de la población, especialmente, de aquellas personas con altos niveles de vulnerabilidad social.
Es imperante la necesidad de una intervención institucional y estatal más eficiente, capaz de intervenir en el modo $y$ en el nivel que la situación exige, capaz de crear otros significados para valores como la justicia y la igualdad, fortaleciendo lazos de solidaridad comunitarios y plasmando una democracia más plena, con el fin de detener el proceso actual de disgregación social que experimentamos. Solo así, tal como lo menciona Galeano (1996), nuestras calles podrán ser menos peligrosas ante nuestros ojos y dejarán de ser prohibidas por la delincuencia o por el pánico a esta; sin embargo, continuarán siendo las calles en donde siempre ocurrirá el peligroso y prodigioso espectáculo de la vida.

\section{BIBLIOGRAFÍA}

Adamson, Gladys. Posmodernidad y la lógica cultural del capitalismo tardío. Argentina: Escuela Argentina de Psicología Social, 1997. [En línea]. En: <http://sincronia.cucsh.udg.mx/ Adamson.htm $>$ [consultado el 30 de abril de 2010].

Baratta, Alesandro. "Derechos Humanos: entre violencia estructural y violencia penal". Revista de Ciencias Jurídicas 68. San José, Costa Rica. Universidad de Costa Rica, 1991: 17-36.

Basaglia, Franco. "Violencia en la marginalidad: el hombre en la Picota". Revista del Instituto de Ciencias Penales y Criminológicas 3 (11). Bogotá, Colombia. Universidad Externado de Colombia, 1980: 43-66.

Bronfebrenner, Urie. La ecología del desarrollo humano. Barcelona, España: Paidós, 2002.

Busso, Gustavo. "Vulnerabilidad social: nociones e implicaciones de políticas para Latinoamérica a inicios del Siglo xxI". Documento presentado en el Seminario Internacional Las Diferentes Expresiones de la Vulnerabilidad Social en América Latina y el Caribe. Santiago de Chile: CEPAL, 20 y 21 de junio de 2001.

Calderón, Rodolfo. "El delito en Costa Rica: una propuesta analítica”. Revista 
Centroamericana en Ciencias Sociales III (1). San José, Costa Rica. Flacso, julio 2006: 83-119.

Cisneros, José Luis. "Cultura, juventud y delincuencia en el Estado de México". Papeles de Población 52. Toluca, México. Universidad Autónoma de México, abriljunio 2007: 255-280.

Claramunt, María Cecilia. "Violencia basada en género y derechos humanos: aproximaciones para trascender el enfoque psicologista de los programas de atención a las víctimas". En Güezmes, Ana y Claramunt, María Cecilia. La violencia contra la mujer: un problema de salud pública y una violación a los derechos humanos. Distrito Nacional, República Dominicana: PRofamilia, 2004.

Fournier, Marco. "Violencia y juventud en América Latina". Revista Nueva Sociedad 167. Argentina, mayo-junio 2000: 147156.

Galeano, Eduardo. "El sacrificio de la justicia en los altares del orden". Revista Reflexiones 48. San José, Costa Rica. Universidad de Costa Rica, julio 1996: 3-9.

Giddens, Anthony. "Estratificación y estructura de clases". En: Giddens, Anthony. Sociología. Madrid, España. Alianza Editorial, 1992: 237-272.

González, María Elena. "Índice de rezago social”. Rosero, Luis (ed.). Costa Rica a la luz del Censo del 2000. San José, Costa Rica. Centro Centroamericano de Población, Universidad de Costa Rica, 2004: 3-27.

Gutiérrez, Miguel. "Exploración de datos censales sobre pobreza aproximada según necesidades básicas insatisfechas (NBI), en la Costa Rica actual". Rosero, Luis (ed.). Costa Rica a la luz del Censo del 2000. San José, Costa Rica. Centro Centroamericano de Población, Universidad de Costa Rica, 2004: 175203.

Hernández, Lang Ying. "Rutas críticas hacia la participación en el narcotráfico de mujeres privadas de libertad en Liberia, Guanacaste". [Tesis de Licenciatura en Psicología]. Guanacaste, Costa Rica: Universidad de Costa Rica, 2011.

Instituto Interamericano de Derechos Humanos. Protección internacional de los derechos económicos, sociales y culturales: Sistema Universal y Sistema Interamericano. San José, Costa Rica: IIDH, 2008.

Juárez, Liliana. "Segregación urbana y sus implicaciones en las ciudades. Una aproximación teórica". Revista de Investigación Científica en Arquitectura (Palapa) 1 (2). Colima, México. Universidad de Colima, julio-diciembre 2006: 45-50.

Martín Baro, Ignacio. "Violencia y agresión social”. En: Martín Baró, Ignacio. Acción e Ideología. El Salvador. UCA Editores, 2000: 359-422.

Organización Panamericana de la Salud. Resumen informe mundial sobre violencia y salud. Washington DC. Organización Mundial de la Salud, 2002: 1-62.

Plaza, Marta. "Sobre el concepto de 'violencia de género'. Violencia simbólica, lenguaje, representación". Extravio. Revista electrónica de literatura comparada 2. España. Universidad de Valencia, 2007: 132-145.

Programa de las Naciones Unidas para el Desarrollo. "Venciendo el temor. Inseguridad ciudadana y desarrollo humano en Costa Rica". Resumen del Informe Nacional de Desarrollo Humano. San José, Costa Rica: PNUD, 2005.

Programa de las Naciones Unidas para el Desarrollo. Informe regional sobre desarrollo humano para América Latina y el Caribe 2010: la desigualdad en Costa Rica. San José, Costa Rica, PNUD: Editorama, 2011.

Quinney, Richard. Una filosofía crítica del orden legal. Venezuela: Órgano de Investigaciones Criminológicas de la Universidad del Zulia, 1975. 
Rodríguez, Francisco. "La pobreza como un proceso de violencia estructural". Revista de Ciencias Sociales x (1). Maracaibo, Venezuela. Universidad del Zulia, eneroabril 2004: 42-50.

Sáenz, Mario. "Algunos aportes para la construcción de una Psicología de la Liberación en Costa Rica: sobre pobreza, desarrollo humano y ética". Revista Costarricense de Psicología 28 (41-45). San José, Costa Rica. El Colegio, 2009: 101-109.

Sebastian, Huhn; Oettler, Anika y Peetz, Peter. "La construcción de realidades inseguras: reflexiones acerca de la violencia en Centroamérica”. Revista de Ciencias Sociales 117-118. Costa Rica. Universidad de Costa Rica, 2007: 73-89.

Sen, Amartya. Commodities and Capabilities. Amsterdam, Países Bajos: North-Holand, 1985.

Sojo, Carlos. "Dimensiones cuantitativas de la pobreza en Costa Rica en la era del Ajuste". En: Sojo, Carlos. Los de en medio: la nueva pobreza en Costa Rica. San José, Costa Rica: FlaCso, 1997.

Fecha de ingreso: 24/05/2011

Fecha de aprobación: 22/11/2011 